\title{
Measuring elastic nonlinearity in a soft solid using a tilted acoustic radiation force for shear wave excitation
}

\author{
Annette Caenen ${ }^{1,2, *}$, Anna E. Knight ${ }^{3}$, Ned C. Rouze ${ }^{3}$, Nick B. Bottenus ${ }^{3}$, Patrick Segers ${ }^{1}$, Kathryn R. Nightingale ${ }^{3}$ \\ ${ }^{1}$ IBiTech-bioMMeda, Ghent University, Ghent, Belgium \\ ${ }^{2}$ Biomedical Engineering, Department of Cardiology, Erasmus MC, University Medical Center Rotterdam, Rotterdam, the Netherlands \\ ${ }^{3}$ Department of Biomedical Engineering, Duke University, Durham, NC, United States \\ *annette.caenen@ugent.be
}

\begin{abstract}
Excitation of multiple wave modes using shear wave elastography can result in additional information about the tissue's material characteristics and, potentially, improve disease diagnosis. Theoretically, tilting the acoustic radiation force excitation axis with respect to the material's symmetry axis should excite several wave modes in the material. In this work, we have experimentally demonstrated proof of concept in a uniaxially stretched phantom, while increasing the stretch level. Tilted acoustic radiation force experiments showed a clearly visible second wave mode across the stretch direction for larger stretches $(>160 \%)$.
\end{abstract}

Keywords-tissue nonlinearity, anisotropy, shear wave elastography, tilting acoustic radiation force, acoustoelasticity, polyvinylalcohol phantoms

\section{INTRODUCTION}

Acoustic radiation force (ARF) based shear wave elastography (SWE) assesses shear wave propagation speed as a measure for the tissue's shear modulus to provide diagnostic information related to diseases affecting soft tissue stiffness. Disease diagnosis could however be improved further by assessing additional soft tissue material properties such as tissue anisotropy or nonlinearity.

It has been proposed that tissue nonlinearity can be assessed based on the theory of acoustoelasticity, which assumes that a nonlinear elastic material exhibits a variation in measured shear wave speed when a uniaxial stress is applied. Gennisson et al. [1] were one of the first to apply this principle in the context of ARF-based SWE in tissue-mimicking phantoms. In their work, the nonlinear shear modulus was estimated in agar-gelatin and polyvinyl alcohol (PVA) phantoms by acquiring SWE measurements perpendicular and parallel to the uniaxial stress direction while gradually increasing the degree of compression. The investigators noted an apparent anisotropy in shear wave speed measurements due to the nonlinear effects of the phantom material, i.e. the speeds measured across the axis of compression were larger than the

This work was supported by the Flemish government agency for Innovation and Entrepreneurship (VLAIO), Research Foundation Flanders (FWO), National Institute of Health (NIH) grants R01-EB022106 and R01CA142824 and NIH Duke Medical Imaging Training Program grant T32 EB001040. estimated speeds along the axis of compression. This observed anisotropy in shear wave speed measurements on stressed PVA phantoms has later been studied by Urban et al. [2] and Chatelin et al. [3] to ratify its use for the study of shear wave propagation in transverse isotropic (TI) materials such as skeletal muscle. The major difference between both studies is that Urban et al. [2] actively applied a stress whereas Chatelin et al. [3] locked in the PVA's anisotropic behavior by uniaxially stretching the phantom during a freeze-thaw cycle in the phantom making process. Recently, the theory of acoustoelasticity has been successfully translated to more clinically relevant applications such as ex vivo kidneys [4] and in vivo breast tissue [5]. These studies used the combination of static elastography and ARF based SWE [6] to estimate the incremental strain and stiffness (shear wave speed) at each stress level.

All previously mentioned SWE experiments focused on the excitation of shear horizontal $(\mathrm{SH})$ wave modes, in which the wave polarization vector is perpendicular to the plane formed by the symmetry axis (fiber or loading axis) and propagation direction. However, it is generally known that more material information can be obtained by exciting multiple wave propagation modes [7]. Indeed, next to the $\mathrm{SH}$ propagation mode, it is desirable to excite the shear vertical (SV) propagation mode - defined by a wave polarization vector parallel to the plane formed by the symmetry axis and

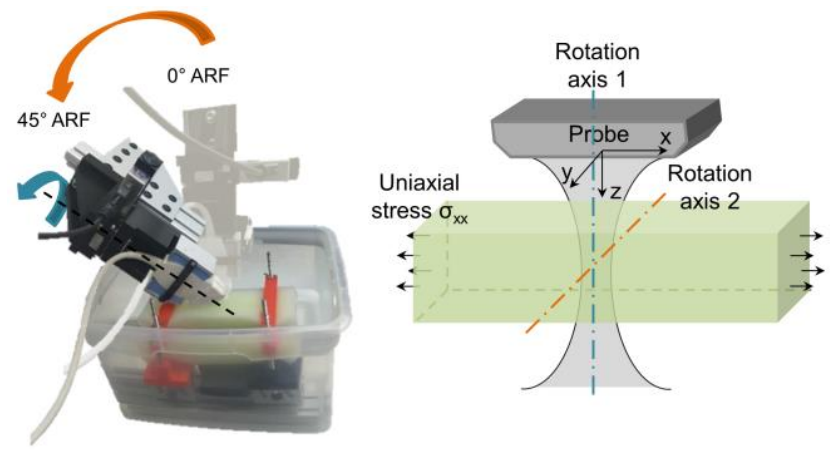

Fig. 1. Experimental set-up of the SWE acquisition demonstrating the two rotation axis of the transducer with respect to the phantom's stretching axis. 

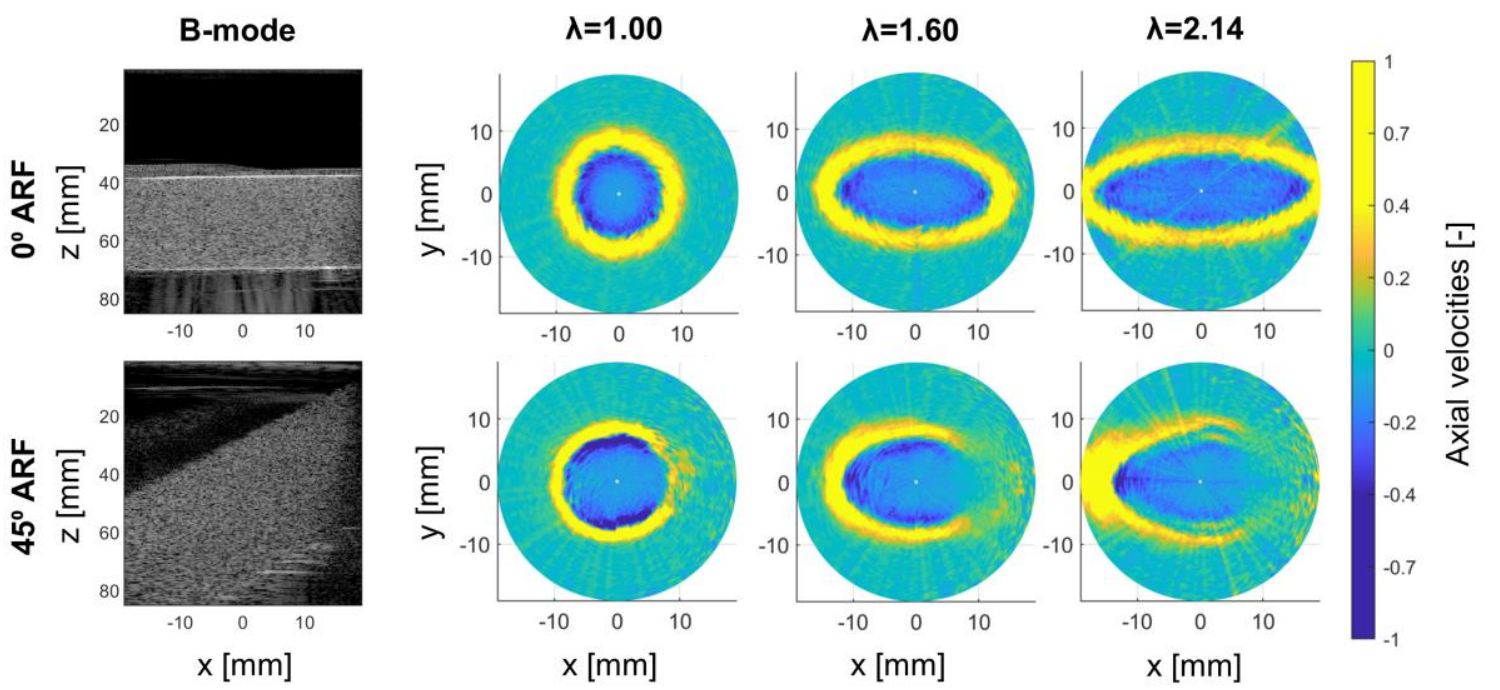

Fig. 2. B-mode along the stretching axis (left) and shear wave propagation in the xy-plane at $2.6 \mathrm{~ms}$ and three different uniaxial stretches $\lambda=1.00,1.60$ and 2.14 (right) for an $\mathrm{ARF}$ tilt of $0^{\circ}$ and $45^{\circ}$ with respect to the stretching axis.

propagation direction. Rouze et al. [7] have shown using theory and simulations that excitation of both SH and SV mode is possible in one SWE acquisition by tilting the ARF with respect to the symmetry axis of the material, but did not perform any actual SWE experiments. The objective of this work is thus to explore experimentally the feasibility to generate and detect both SV and SH propagation modes using ARF-based SWE in a soft tissue mimicking medium with a symmetry axis. In this study, we describe an experimental setup that allows rotation of the ARF with respect to the material's symmetry axis and describe measurements in stretched phantoms to extend previous experiments in [1-3].

\section{MATERIALS AND Methods}

\section{A. PVA phantom with symmetry axis}

Tissue-mimicking material was created by heating a mixture of 8\% PVA (PVA 98-99\% hydrolyzed, Alfa Aesar, Ward Hill, MA, USA), 1\% cellulose (Sigmacell Cellulose $20 \mu \mathrm{m}$, Sigma Life Science, Darmstadt, Germany), 50.4\% coolant (Concentrate Antifreeze, Prestone, Chicago, IL, USA) and $33.6 \%$ deionized water. After cooling to room temperature, the $8 \%$ PVA solution was poured into a beam-shaped mold $(50 \times 50 \times 200 \mathrm{~mm})$ and freeze-thawed once [8]. The phantom was subsequently subjected to a uniaxial stretch $\lambda$ along the $x-$ direction (see Fig. 1), varying from 1.0 to 2.0 in steps of 0.2 , with a final maximum stretch of 2.14 .

\section{B. SWE acquisition with ARF tilt}

The L7-4 probe connected to the Vantage 256 research system (Verasonics, Kirkland, WA, USA) was mounted on a mechanical arm that enabled two kinds of rotations: (i) rotation around the transducer's axis (rotation axis 1 or z-axis in Fig. 1) to perform 3D SWE measurements and (ii) rotation around the axis perpendicular to the plane formed by the phantom's stretching axis and transducer's axis (rotation axis 2 or y-axis in Fig. 1) to tilt the ARF from $0^{\circ}$ to $45^{\circ}$ with respect to the stretching axis. For each stretch level and ARF tilt, 3D SWE measurements were performed by rotating the transducer around its axis in steps of $2.5^{\circ}$. Shear waves were generated using a $4 \mathrm{MHz}$ push for $300 \mu \mathrm{s}$. Shear wave imaging employed plane waves in combination with coherent compounding $\left(-3^{\circ}\right.$, $0^{\circ}, 3^{\circ}$ ) for $20 \mathrm{~ms}$, which resulted in an effective frame rate of about $5 \mathrm{kHz}$ [9].

Axial particle velocity motion was estimated according to Kasai's autocorrelation technique [9]. A smaller spatial temporal domain of the axial velocities at around the axial pushing focus (averaged over a depth-of-field of $1 \mathrm{~mm}$ ) was selected to determine the wave speed using the Radon sum transformation [10]. All SWE post-processing was performed in Matlab R2018b (The MathWorks Inc., Natick, MA, USA).

\section{RESULTS}

The acoustoelastic effect is demonstrated in the upper row of images in Fig. 2, where it is observed that the shear wave propagation pattern in the xy-plane changes from a circle when no stretch is applied - to an ellipse - when a stretch is applied. Tilting the ARF from $0^{\circ}$ to $45^{\circ}$ gave rise to similar wave patterns for stretches 1.00 and 1.60 , as displayed in the second row of images in Fig. 2. Differences in tissue velocity amplitude are however noticeable for positive $\mathrm{x}$-coordinates due to the increasing acoustic attenuation by the additional phantom material when the probe is tilted (see B-modes in Fig.2). However, the wave propagation pattern for a stretch $\lambda$
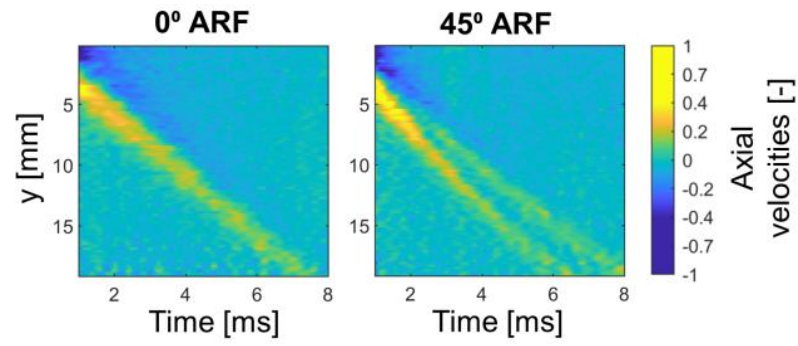

Fig. 3. Observation of shear wave splitting along the y-direction in a uniaxially stretched phantom $(\lambda=2.14)$ when tilting the ARF $45^{\circ}$ with respect to the stretching axis. 


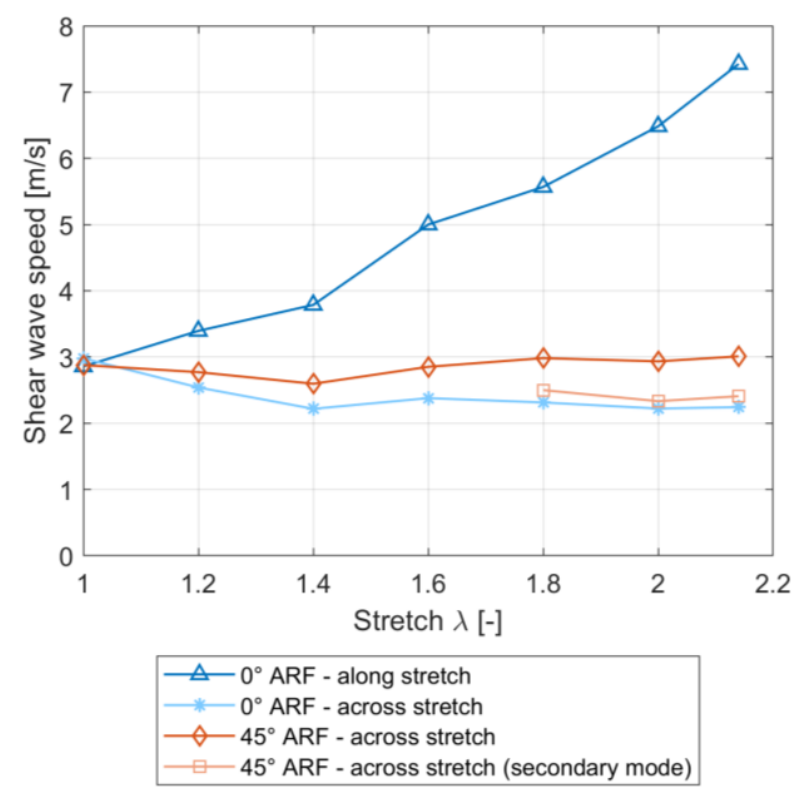

Fig. 4. Shear wave speeds as a function of stretch for $0^{\circ} \mathrm{ARF}$ tilt (along and across the stretch direction) and for $45^{\circ}$ ARF tilt (across the stretch direction).

of 2.14 and $45^{\circ} \mathrm{ARF}$ tilt exhibited two new wave features (see right bottom panel of Fig. 2): (i) cusp-like patterns are observed along the stretching axis (semi-major ellipse axis) and (ii) a second wave mode appeared across the stretching direction (semi-minor ellipse axis). The shear wave splitting across the stretching direction becomes more obvious when we plot the axial tissue velocities as a function of the $y$-direction (semi-minor ellipse axis) and time, as can be seen in Fig. 3.

The shear wave speeds are determined along and across the stretching axis for each considered stretch level in Fig. 4. For $0^{\circ}$ ARF tilt, the wave speeds varied between 2.8 and $7.4 \mathrm{~m} / \mathrm{s}$ along the stretching axis and between 3.0 and $2.1 \mathrm{~m} / \mathrm{s}$ across the stretching axis for increasing stretch $(\lambda=1.00-2.14)$. For $45^{\circ}$ ARF tilt and across the stretching direction, one clear wave mode was detected with a speed of 2.6-2.9 m/s for stretches $\lambda=1.00-1.60$. In the same SWE set-up, two wave modes were visible for stretches $\lambda=1.80-2.14$, one with a speed of about 3.0 $\mathrm{m} / \mathrm{s}$ and the other with a speed between $2.3-2.5 \mathrm{~m} / \mathrm{s}$.

\section{DisCUSSION AND CONCLUSION}

This work demonstrated the feasibility to experimentally excite multiple wave modes in a tissue-mimicking phantom with one symmetry axis using a tilted ARF set-up in SWE. The material symmetry axis was induced by applying a uniaxial stretch to the phantom (so-called acoustoelastic effect).

The acoustoelastic effect has been studied before in the context of ARF-based SWE resulting in SH-mode excitation [1-3], but not with a tilted ARF set-up. Tilting the ARF $45^{\circ}$ with respect to the stretching direction resulted in a higher shear wave speed across the stretching direction (as can be clearly seen in Fig. 2 and 4). At high stretches $(\lambda>1.60)$, a second wave mode appeared across the stretching direction of which the speed corresponded to the speed measured with no
ARF tilt. This suggests that the mode excited across the stretch could correspond to the SV-mode [7]. Currently, finite element simulations and theoretical derivations are ongoing to better understand the excited shear wave physics.

When interpreting these results, it should be kept in mind that there are some study limitations. The increasing acoustic attenuation created some additional challenges in shear wave visibility and speed estimation for a part of the excited wave (see Fig. 2). Additionally, it should be taken into account that the applied stress might not be perfectly uniaxially oriented, especially closer to the clamps.

In conclusion, this study showed that a tilted ARF set-up in a soft solid with one symmetry axis induces multiple wave modes that can be used to observe more information about wave propagation than the conventionally used $0^{\circ} \mathrm{ARF}$ set-up. These types of wave measurements can thus provide additional information about the material models of stretched PVA, and potentially about tissue nonlinearity or transverse anisotropy in skeletal muscle.

\section{REFERENCES}

[1] J. L. Gennisson, M. Renier, S. Catheline, C. Barriere, J. Bercoff, M Tanter and M. Fink, "Acoustoelasticity in Soft Solids: Assessment of the Nonlinear Shear Modulus with the Acoustic Radiation Force," J. Acoust. Soc. Am., vol. 116, pp. 3211-3219, Dec. 2007.

[2] M. W. Urban, M. Lopera, S. Aritizabal, C. Amador, I. Nenadic, R. R. Kinnick, A. D. Weston, B. Qiang, X. Zhang, J. F. Greenleaf, "Characterization of Transverse Isotropy in Compressed TissueMimicking Phantoms," IEEE Trans. Ultrason. Ferroelectr. Freq. Control, vol. 62, pp. 1036-1046, June 2015.

[3] S. Chatelin, M. Bernal, T. Deffieux, C. Papadacci, P. Flaud, A. Nahas, C. Boccara, J.-1. Gennisson, M. Tanter, M. Pernot, "Anisotropic polyvinyl alcohol hydrogel phantom for shear wave elastography in fibrous bological soft tissue: a multimodality characterization," Phys. Med. Biol., vol 59, pp. 6923-6940, 2014.

[4] S. Aristizabal, C. A. Carrascal, I. Z. Nenadic, J F. Greenleaf, M. W. Urban, "Application of Acoustoelasticity to Evaluate Nonlinear Modulus in Ex Vivo Kidneys," IEEE Trans. Ultrason. Ferroelectr. Freq. Control, vol. 65, pp. 188-200, Feb 2018

[5] M. Bernal, F. Chamming's, M. Couade, J. Bercoff, M. Tanter, and J. L. Gennisson, "In Vivo Quantification of the Nonlinear Shear Modulus in Breast Lesions: Feasibility Study," IEEE Trans. Ultrason. Ferroelectr. Freq. Control, vol. 63, pp. 101-9, Jan 2016.

[6] H. Latorre-Ossa, J.-L. Gennisson, E. De Brosses, M. Tanter, "Quantitative imaging of nonlinear shear modulus by combining static elastography and shear wave elastography," IEEE Trans. Ultrason. Ferroelectr. Freq. Control, vol. 59, pp. 833-839, April 2012.

[7] N. C. Rouze, M. H. Wang, M. L. Palmeri, and K. R. Nightingale, "Finite element modeling of impulsive excitation and shear wave propagation in an incompressible, transversely isotropic medium," J. Biomech., vol. 46, pp. 2761-8, Nov 2013.

[8] K. J. M. Surry, H. J. B. Austin, A. Fenster, T. M. Peters, "Poly(vinyl alochol) cryogel phantoms for use in ultrasound and MR imaging," Phys. Med. Biol., vol. 49, pp. 5529-5546, 2004.

[9] Y. Deng, N. C. Rouze, M. L. Palmeri, K. R. Nightingale, "Ultrasonic shear wave imaging sequencing and data processing using a Verasonics research scanner,'IEEE Trans. Ultrason. Ferroelectr. Freq. Control, vol. 64, pp. 164-176, Jan 2017.

[10] N. C. Rouze, M. H. Wang, M. L. Palmeri, K. R. Nightingale, "Robust estimation of time-of-flight shear wave speed using a Radon Sum transformation," IEEE Trans. Ultrason. Ferroelectr. Freq. Control, vol. 57, pp. 2662-2670, Dec 2010. 